\title{
Correlated mean field Ansatz for the Kondo necklace
}

\author{
H. Y. Kee* and P. Fazekas ${ }^{\dagger}$ \\ International Centre for Theoretical Physics, \\ P.O. Box 586, I-34100 Trieste, Italy
}

\begin{abstract}
We study the ground state phase diagram of the pseudospin model introduced by Doniach to describe the essential physics of Kondo lattices. We use variational trial states which augment the usual mean field solution by incorporating various intersite correlations. A composite spin correlation describing the antiparallel alignment of fluctuating triplets is found to be particularly favourable for large Kondo couplings. With this trial state, the magnetic-to-Kondo transition is suppressed and the strong coupling ground state is ordered with strongly reduced moments. The relevance of the findings is discussed.
\end{abstract}

*Permanent address: Department of Physics Education, Seoul National Unversity, Seoul, 151-742 Korea

${ }^{\dagger}$ Permanent address: Research Institute for Solid State Physics, P.O.B. 49, Budapest 114, H-1525 Hungary 


\section{The model}

The basic question in the physics of heavy fermion materials is whether collective spin compensation can be taking place in a periodic array of localized moments immersed in a conduction electron sea [1]. In case yes, we may speak about a collective Kondo effect even though it remains unclear to which extent the formation of an overall lattice singlet ground state can be likened to the single-ion Kondo effect. The most intriguing possibility is that spin compensation may go a very long way before it is stopped by the ordering of the residual tiny moments [2].

As far as spins are concerned, the Kondo effect is just a spin compensation phenomenon. The emergence of a non-analytic energy scale in the impurity problem is connected with the existence of a large number of arbitrarily lowlying electron-hole excitations. Though variational methods indicate the existence of a lattice-coherence-enhanced Kondo energy scale for the nearly integral valent (Kondo) regime of the Anderson lattice [3, 4], as well as for the Kondo lattice [5, 6], they do not provide a proof that the ground state energy of the Kondo lattice contains non-analytic terms. The interplay of spin and charge degrees of freedom in the Kondo lattice may still prove to be quite different from what one has found for the impurity problem. It seems desirable to separate, if possible, the spin compensation aspect from all the other complications of "true Kondo physics".

It has become accepted [7, 8] that the competition of spin compensation and magnetic ordering can be described with drastically simplified models which contain just the spin degrees of freedom. The simplest of these is the Kondo necklace model introduced by Doniach [9]. In addition to the localized spins $\vec{S}$ of the $f$-electrons we introduce a set of pseudospins $\vec{\tau}$ 's which stand for the spin degrees of freedom of the conduction electrons. The number of $\vec{S}$-spins is chosen equal to the number of $\vec{\tau}$ spins which implies that the possibility of a full spin compensation is a feature of the system. While it can be argued [5, 10] that a singlet ground state may arise at any band filling, it is more straightforward to associate the model with the Kondo lattice with a half-filled conduction band so that the number of pseudospins is equal to the number of conduction electrons. This has the additional motivation that the corresponding Kondo lattice has an insulating ground state [1]; the appearance of a charge gap is a justification for omitting the charge degrees of freedom. 
The simplest mean field treatment of the original necklace model yielded the beautiful result of a ground state phase transition from a magnetically ordered to a fully spin-compensated state [9]. We are by now fully aware that strictly one-dimensional models are bound to show much subtler behaviour [0] but extending the model to the physically more relevant higher dimensions (no longer "necklaces" in the geometrical sense) makes us to expect that the mean field results are roughly correct.

Our intention here is to improve the mean field approximation by including short-range correlations. Such an approach is expected to give sensible improvements over single-site mean field results in three dimensions where the mean field phase diagram should be qualitatively correct. However, intermediate steps of our calculation can be executed free of further approximations in one dimension. Therefore, we make the algebra (sum over local configurations) for the one-dimensional case but bear in mind that the character of the results is meant for three dimensions. (In one dimension, the better estimate of the ground state energy is still believable but one should not trust the characterization of the ground state.)

We study $S=1 / 2$ Kondo necklace models

$$
H=J \sum_{i=1}^{L} \vec{S}_{i} \cdot \vec{\tau}_{i}+W \sum_{i=1}^{L}\left(\tau_{i}^{x} \tau_{i+1}^{x}+\tau_{i}^{y} \tau_{i+1}^{y}+\rho \tau_{i}^{z} \tau_{i+1}^{z}\right)
$$

The necklace is closed with the periodic boundary condition $L+1 \equiv 1$.

Seeking correspondence with the insulating state of the Kondo lattice model would lead us to choose an antiferromagnetic Kondo coupling $J>0$, and an antiferromagnetic intersite pseudospin coupling $W>0$. Actually, in Doniach's [9] original pseudospin model, the latter term was chosen to be purely $x-y$-like $(\rho=0)$, which gives a good imitation of propagating degrees of freedom. One should remember, however, that the underlying fermionic Kondo lattice problem had spin-rotational symmetry, and this has been lost by postulating the $x-y$ form of coupling. We note that isotropic spin models (or anisotropic models with isotropy as a special case) have been discussed in the literature with the purpose of modelling Kondo lattice physics [12, 4]. In any case, a more complete understanding of the spin system makes the study of an extended model desirable. With this motivation, the study of (11) with arbitrary signs of $J$ and $W$, and with a general anisotropy $0 \leq \rho<\infty$ is indicated. For reasons of convenience, we concentrate on the case $\rho=0$ 
but we wish to emphasize that the method used here is equally applicable for $\rho \neq 0$.

Hamiltonians of the form (四) can be defined for either $|\vec{S}|>|\vec{\tau}|$, or $|\vec{\tau}|>|\vec{S}|$, or $|\vec{\tau}|=|\vec{S}|$, corresponding to underscreened, overscreened, or exactly screened Kondo lattices. Generalizing Doniach's work on the exactly screened $S=1 / 2$ model, we have discussed the mean field ground states of the underscreened [13] and overscreened [14], Kondo necklace models earlier. Here our main interest lies in going beyond the single-site mean field description, therefore we confine our attention to the simplest case $|\vec{S}|=1 / 2$, and $|\vec{\tau}|=1 / 2$. The Hilbert space of a lattice site is spanned by the four local basis states $\left|S^{z} \tau^{z}\right\rangle$

$$
\begin{aligned}
|1\rangle=|1 / 2,1 / 2\rangle & |2\rangle & =|-1 / 2,1 / 2\rangle \\
|3\rangle=|1 / 2,-1 / 2\rangle & |4\rangle & =|-1 / 2,-1 / 2\rangle
\end{aligned}
$$

The number of cases is reduced if we set $\rho=0$. It is wellknown that the ferromagnetic and antiferromagnetic $S=1 / 2 x-y$ models (on bipartite lattices) are physically identical since they can be connected by a canonical transformation. A bipartite lattice can be divided into alternate sublattices $A$ and $B$ so that nearest-neighbour bonds always connect different sublattices. Then the transformation

$$
\hat{U}_{1}=\prod_{j \in B} \exp \left(-i \pi \tau_{j}^{z}\right)
$$

changes the sign of the $x-y$ term

$$
\hat{U}_{1}\left(W \sum_{i=1}^{L}\left(\tau_{i}^{x} \tau_{i+1}^{x}+\tau_{i}^{y} \tau_{i+1}^{y}\right)\right) \hat{U}_{1}^{-1}=-W \sum_{i=1}^{L}\left(\tau_{i}^{x} \tau_{i+1}^{x}+\tau_{i}^{y} \tau_{i+1}^{y}\right)
$$

A similar statement holds for our model: a $\pi$-rotation about the spin- $z$ axes for both the $S^{-}$, and $\tau$-spins on sublattice $B$

$$
\hat{U}_{2}=\prod_{j \in B} \exp \left[-i \pi\left(S_{j}^{z}+\tau_{j}^{z}\right)\right]
$$

changes the sign of the intersite $\tau$-coupling while leaving the Kondo term unchanged

$$
\hat{U}_{2}\left(J \sum_{i=1}^{L} \vec{S}_{i} \cdot \vec{\tau}_{i}+W \sum_{i=1}^{L}\left(\tau_{i}^{x} \tau_{i+1}^{x}+\tau_{i}^{y} \tau_{i+1}^{y}\right)\right) \hat{U}_{2}^{-1}
$$




$$
=J \sum_{i=1}^{L} \vec{S}_{i} \cdot \vec{\tau}_{i}-W \sum_{i=1}^{L}\left(\tau_{i}^{x} \tau_{i+1}^{x}+\tau_{i}^{y} \tau_{i+1}^{y}\right)
$$

The second term on the right-hand side can be transformed to a noninteracting spinless fermion model [15]: for half-filling, the ground state energy is $-W / \pi$. This provides a useful comparison for energy estimates in the weak $-J$ regime.

The inclusion of $\rho \neq 0$ would, of course, make the cases of positive and negative $W$ genuinely different. Our variational method is, in principle, just as applicable for $\rho \neq 0$ as for $\rho=0$. Restricting our attention to the case $\rho=0$, where it is sufficient to consider $W<0$, is motivated by formal convenience: for ferromagnetic intersite coupling, homogeneous trial states can be used.

\section{Variational method}

We wish to map out the ground state phase diagram of $H$. For this purpose, we introduce variational trial states. Since a site $i$ has two spins, the "true" spin $\vec{S}_{i}$, and the pseudospin $\vec{\tau}_{i}$ which are coupled by the Kondo term, we can speak of the internal structure of the site, which is described as a linear combination of the four possible states

$$
|\phi\rangle_{i}=\alpha_{1}|1\rangle_{i}+\alpha_{2}|2\rangle_{i}+\alpha_{3}|3\rangle_{i}+\alpha_{4}|4\rangle_{i}
$$

In the single-site mean field theory, the internal state of the site $i$ is taken to be independent of the instantaneous state of any other site; e.g., a translationally invariant state would be described by the product wave function

$$
|\Phi\rangle=\prod_{i=1}^{L}|\phi\rangle_{i}=\prod_{i=1}^{L}\left(\alpha_{1}|1\rangle_{i}+\alpha_{2}|2\rangle_{i}+\alpha_{3}|3\rangle_{i}+\alpha_{4}|4\rangle_{i}\right)
$$

In a ground state with antiferromagnetic long-range order, $|\phi\rangle_{i}$ can be made sublattice-dependent

$$
|\Phi\rangle_{A F}=\prod_{i=1}^{L / 2}\left|\phi^{A}\right\rangle_{2 i-1}\left|\phi^{B}\right\rangle_{2 i}
$$




$$
\begin{aligned}
= & \prod_{i=1}^{L / 2}\left(\alpha_{1}^{A}|1\rangle_{2 i-1}+\alpha_{2}^{A}|2\rangle_{2 i-1}+\alpha_{3}^{A}|3\rangle_{2 i-1}+\alpha_{4}^{A}|4\rangle_{2 i-1}\right) . \\
& \left(\alpha_{1}^{B}|1\rangle_{2 i}+\alpha_{2}^{B}|2\rangle_{2 i}+\alpha_{3}^{B}|3\rangle_{2 i}+\alpha_{4}^{B}|4\rangle_{2 i}\right)
\end{aligned}
$$

but otherwise, the simple product form is retained.

We propose to improve the variational description by including nearestneighbour intersite correlations, according to the recipe: if site $i$ is in the state $\left|\xi_{i}\right\rangle_{i}$, and site $i+1$ in the state $\left|\xi_{i+1}\right\rangle_{i+1}$, then the amplitude acquires the additional factor $O\left(\xi_{i}, \xi_{i+1}\right)$. For a translationally invariant state, we can write

$$
|\Psi\rangle=\prod_{i} \hat{P}_{i, i+1}|\Phi\rangle
$$

where the intersite correlator

$$
\hat{P}_{i, i+1}=\sum_{\xi_{i}=1}^{4} \sum_{\xi_{i+1}=1}^{4}\left|\xi_{i}\right\rangle_{i}\left|\xi_{i+1}\right\rangle_{i+1} O\left(\xi_{i}, \xi_{i+1}\right)_{i+1}\left\langle\xi_{i+1}\right|{ }_{i}\left\langle\xi_{i}\right|
$$

has been introduced.

The general structure of the variational trial state is that it is created by an intersite projection operator $\hat{P}$ acting on a mean-field reference state $|\Phi\rangle$. On-site correlations (such as local singlet formation) are included in $|\Phi\rangle$, and intersite correlations are controlled by $\hat{P}$. Both $|\Phi\rangle$ and $\hat{P}$ can contain variational parameters.

Analogous trial states could be written down for higher-dimensional lattices. Actually, these would be physically more acceptable: our variational method is a correlated mean field method. Thus the overall appearance of our results is what we would expect for three-dimensional systems. Subtle features particular to one dimension [7] are likely to be missed by the present treatment. However, the algebra needed for the variational method can be executed fully in one dimension while it would involve further approximations in higher dimensions. Therefore we stick to the one-dimensional case anticipating that care has to be exercised in interpreting the findings.

The method we use has been introduced for spin chains by Virosztek [16]; later it was applied to the one-dimensional Hubbard model by Penc and one of us [17].

We will be working with homogeneous states for which

$$
\sum_{i}\left(S_{i}^{z}+\tau_{i}^{z}\right)=0
$$


The motivation comes from considering the single-site mean-field solution [9] of the original necklace model $W>0, J>0, \rho=0$. In the simplest approximation, the ground state is an $x-y$ antiferromagnet if $J<W$, and a collection of independent singlets if $J>W$, with a continuous phase transition at $J=W$. The finding of a ground state phase transition between a magnetic and a "Kondo-like" state has long been the source of inspiration for continuing research in the Kondo lattice physics. On the other hand, it has been a matter of debate whether this transition is an exact consequence of the model (11), or an artefact of the simple approximation. At the simplest level, one can point it out that the description of the non-ordered state as strictly singlet is just a zeroth-order approximation since for any finite $W / J$, however small, the process

$$
|1 / 2,-1 / 2\rangle_{1}|-1 / 2,1 / 2\rangle_{2} \longrightarrow|1 / 2,1 / 2\rangle_{1}|-1 / 2,-1 / 2\rangle_{2}
$$

will mix in local triplets.

It is natural to expect that the description in terms of local singlets becomes correct only in the limit $J / W \rightarrow \infty$, and for finite $J$, there will be spin-spin correlations between the sites. It is an interesting question whether the mean-field transition survives the inclusion of such correlations.

\section{Variational trial states for the necklace model}

\subsection{Homogeneous states}

In accordance with (12), in (8) we choose

$$
\left|\alpha_{1}\right|=\left|\alpha_{4}\right|=\alpha \quad \text { and } \quad\left|\alpha_{2}\right|=\left|\alpha_{3}\right|=\beta
$$

One still has to decide the relative phase factors.

The ground state wave function can be chosen as real, which says all the parameters are real. The remaining task is to specify the relative signs of the $\alpha$ 's. This we do by requiring that non-diagonal processes should give a negative contribution to the energy, whenever that is possible. The on-site Kondo spin-flip term involves the factor $\alpha_{2} \alpha_{3}$; the term can be made negative if

$$
\operatorname{sg}\left(\alpha_{2} \alpha_{3}\right)=-\operatorname{sg}(J)
$$


As we are going to see, there are two kinds of contributions coming from the intersite $\tau$-spin-flip processes. One of them, which acts between sites with antiparallel $S$-spins, was illustrated in (13). Here all four local states appear once, so the term comes with the factor $\alpha_{1} \alpha_{2} \alpha_{3} \alpha_{4}$. The corresponding energy term can be made negative if we prescribe

$$
\operatorname{sg}\left(\alpha_{1} \alpha_{4}\right)=\operatorname{sg}(J) \cdot \operatorname{sg}(W)
$$

In the other kind of hopping process, the $S$-spins are parallel

$$
|1 / 2,1 / 2\rangle_{1}|1 / 2,-1 / 2\rangle_{2} \longrightarrow|1 / 2,-1 / 2\rangle_{1}|1 / 2,1 / 2\rangle_{2}
$$

In the corresponding term, all variational parameters are raised to even powers, so the sign of the contribution is the same as the sign of $W$. In particular, for $W>0$, a homogeneous Ansatz does not permit to gain energy from this kind of process.

No difficulties arise if we stick to the case $\rho=0$ : In (6) we have shown that $W>0$ and $W<0$ are equivalent, so we can choose $W<0$, and have all contributions negative. Still, it is interesting to remember that the equivalent solution of the $W>0$ problem is a two-sublattice antiferromagnetic state which can be generated from the homogeneous Ansatz by acting on it with the transformation $\hat{U}_{2}$ given in (司)

$$
\begin{aligned}
\hat{U}_{2} \cdot \prod_{i=1}^{L}\left(\alpha|1\rangle_{i}-\beta|2\rangle_{i}+\beta|3\rangle_{i}-\alpha|4\rangle_{i}\right) \\
=\prod_{i=1}^{L / 2}\left(\alpha|1\rangle_{2 i-1}-\beta|2\rangle_{2 i-1}+\beta|3\rangle_{2 i-1}-\alpha|4\rangle_{2 i-1}\right) \\
\quad\left(\alpha|1\rangle_{2 i}+\beta|2\rangle_{2 i}-\beta|3\rangle_{2 i}-\alpha|4\rangle_{2 i}\right)
\end{aligned}
$$

The two-sublattice form is just Doniach's [9] Ansatz for the case $W>0$.

Henceforth we keep $W<0$. Depending on the sign of $J$, there are two different classes of wave functions. To be specific, we choose $J>0$. The Ansatz for the ground state reads

$$
|\Psi\rangle=\prod_{i} \hat{P}_{i, i+1}\left(\alpha|1\rangle_{i}+\beta|2\rangle_{i}-\beta|3\rangle_{i}+\alpha|4\rangle_{i}\right)
$$


The structure of the correlator can be represented by the matrix

$$
\hat{O}=\left(\begin{array}{cccc}
O\left(\xi_{1}, \xi_{1}\right) & O\left(\xi_{1}, \xi_{2}\right) & O\left(\xi_{1}, \xi_{3}\right) & O\left(\xi_{1}, \xi_{4}\right) \\
O\left(\xi_{2}, \xi_{1}\right) & O\left(\xi_{2}, \xi_{2}\right) & O\left(\xi_{2}, \xi_{3}\right) & O\left(\xi_{2}, \xi_{4}\right) \\
O\left(\xi_{3}, \xi_{1}\right) & O\left(\xi_{3}, \xi_{2}\right) & O\left(\xi_{3}, \xi_{3}\right) & O\left(\xi_{3}, \xi_{4}\right) \\
O\left(\xi_{4}, \xi_{1}\right) & O\left(\xi_{4}, \xi_{2}\right) & O\left(\xi_{4}, \xi_{3}\right) & O\left(\xi_{4}, \xi_{4}\right)
\end{array}\right)
$$

Considering the obvious symmetry

$$
O\left(\xi_{i}, \xi_{j}\right)=O\left(\xi_{j}, \xi_{i}\right)
$$

and further restrictions arising from $\uparrow-\downarrow$ symmetry, still leaves us with more variational parameters than one could easily handle. One has to try to guess what are the relevant correlations. We return to this question later. Before doing that, we outline the general formalism.

\subsection{Transfer matrix formalism}

We have to calculate the ground state energy

$$
E=\frac{\langle\Psi|H| \Psi\rangle}{\langle\Psi \mid \Psi\rangle}
$$

and minimize it with respect to the variational parameters.

Let us keep the matrix $\hat{O}$ and the choice of the $\alpha$ 's as yet unspecified and calculate the norm $\langle\Psi \mid \Psi\rangle$. Expanding $|\Psi\rangle$ in the orthonormal basis formed as the direct product of the single-site bases (2)

$$
|\Psi\rangle=\sum_{\xi_{1}=1}^{4} \sum_{\xi_{2}} \ldots \sum_{\xi_{L}} \prod_{i=1}^{L}\left(\alpha_{\xi_{i}} O\left(\xi_{i}, \xi_{i+1}\right)\right)\left|\xi_{1}\right\rangle_{1}\left|\xi_{2}\right\rangle_{2} \ldots\left|\xi_{L}\right\rangle_{L}
$$

we find that the norm is given by a sum over configurations, which is similar to the partition function of a one-dimensional classical lattice model. It is advantageous to introduce the transfer matrix $\hat{T}$ as

$$
T\left(\xi_{i}, \xi_{i+1}\right)=\left|\alpha_{\xi_{i}}\right| O^{2}\left(\xi_{i}, \xi_{i+1}\right)\left|\alpha_{\xi_{i+1}}\right|
$$


whereupon the norm becomes

$$
\begin{aligned}
\langle\Psi \mid \Psi\rangle & =\sum_{\xi_{1}} \sum_{\xi_{2}} \ldots \sum_{\xi_{L}} T\left(\xi_{1}, \xi_{2}\right) T\left(\xi_{2}, \xi_{3}\right) \ldots T\left(\xi_{L-1}, \xi_{L}\right) T\left(\xi_{L}, \xi_{1}\right) \\
& =\operatorname{Tr}\left(\hat{T}^{L}\right) \longrightarrow x_{0}^{L}
\end{aligned}
$$

where $x_{0}$ is the largest eigenvalue of the transfer matrix.

The next step is the calculation of expectation values. The knowledge of $x_{0}$ suffices to determine the densities of quantities which are diagonal in the representation (2). The density of sites in the state $|\xi\rangle$ is

$$
n_{\xi}=\alpha_{\xi}^{2} \frac{\partial \ln x_{0}}{\partial \alpha_{\xi}^{2}}
$$

The combined density of nearest-neighbour pairs in the configuration $\left|\xi_{1}\right\rangle\left|\xi_{2}\right\rangle$, and its reverse $\left|\xi_{1}\right\rangle\left|\xi_{2}\right\rangle$ is

$$
n_{\xi_{1} \xi_{2}}=O^{2}\left(\xi_{1}, \xi_{2}\right) \frac{\partial \ln x_{0}}{\partial O^{2}\left(\xi_{1}, \xi_{2}\right)}
$$

where the symmetry of the matrix $\hat{O}$ was exploited.

The straightforward analogy with classical statistical mechanics ceases when we go over to the calculation of off-diagonal quantities such as spinflip amplitudes. At this stage, it becomes apparent that we are dealing with a genuinely quantum-mechanical problem. We follow the method introduced by Virosztek [16.

Let us illustrate the method on the example of the spin-flip part of the Kondo term acting at site $m$

$$
\begin{aligned}
& \left\langle\Psi\left|S_{m}^{+} \tau_{m}^{-}+S_{m}^{-} \tau_{m}^{+}\right| \Psi\right\rangle= \\
& \sum_{\xi_{1}} \ldots \sum_{\xi_{m-1}} \sum_{\xi_{m}} \sum_{\xi_{m}^{\prime}} \sum_{\xi_{m+1}} \ldots \sum_{\xi_{L}} \prod_{i=1}^{m-1} \alpha_{\xi_{i}}^{2} \cdot \prod_{i=1}^{m-2} O^{2}\left(\xi_{i}, \xi_{i+1}\right) \\
& \cdot \alpha_{\xi_{m}} O\left(\xi_{m-1}, \xi_{m}\right) O\left(\xi_{m}, \xi_{m+1}\right)\left\langle\xi_{m}\left|S_{m}^{+} \tau_{m}^{-}+S_{m}^{-} \tau_{m}^{+}\right| \xi_{m}^{\prime}\right\rangle \\
& \cdot \alpha_{\xi_{m}^{\prime}} O\left(\xi_{m-1}, \xi_{m}^{\prime}\right) O\left(\xi_{m}^{\prime}, \xi_{m+1}\right) \cdot \prod_{i=m+1}^{L}\left(\alpha_{\xi_{i}}^{2} O^{2}\left(\xi_{i}, \xi_{i+1}\right)\right)
\end{aligned}
$$

The expression is rather like the norm (25) except that at site $m$, a disturbance has occured which, via the correlators, influences the sites $m-1$ and 
$m+1$. (28) is still the trace of a product of matrices; however, in contrast

to (25), not all matrices are $\hat{T}$. The sites $m-1$ and $m+1$ are connected by the matrix $\hat{K}_{ \pm}$rather than by $\hat{T}^{2}$

$$
\left\langle\Psi\left|S_{m}^{+} \tau_{m}^{-}+S_{m}^{-} \tau_{m}^{+}\right| \Psi\right\rangle=\operatorname{Tr}\left(\hat{T}^{m-2} \hat{K}_{ \pm} \hat{T}^{L-m}\right)
$$

Doing the sums over $\xi_{m}$ and $\xi_{m}^{\prime}$ we recall that the spin-flip terms connect the states $|2\rangle$ and $|3\rangle$. It can be read off from (28) that

$$
\begin{aligned}
K_{ \pm}\left(\xi_{m-1}, \xi_{m+1}\right)= & 2\left|\alpha_{\xi_{m-1}} \alpha_{\xi_{m+1}}\right| \alpha_{2} \alpha_{3} \\
& \cdot O\left(\xi_{m-1}, 2\right) O\left(2, \xi_{m+1}\right) O\left(\xi_{m-1}, 3\right) O\left(3, \xi_{m+1}\right)
\end{aligned}
$$

The exponential dominance of the trace by factors of $x_{0}$ allows to deduce

$$
\frac{\left\langle\Psi\left|S_{m}^{+} \tau_{m}^{-}+S_{m}^{-} \tau_{m}^{+}\right| \Psi\right\rangle}{\langle\Psi \mid \Psi\rangle}=\frac{\left\langle X_{0}\left|\hat{K}_{ \pm}\right| X_{0}\right\rangle}{x_{0}^{2}}
$$

where $\left|X_{0}\right\rangle$ is the eigenvector satisfying $\hat{T}\left|X_{0}\right\rangle=x_{0}\left|X_{0}\right\rangle$. Because of the symmetry of $\hat{T}$, it is of the form

$$
\left|X_{0}\right\rangle=\frac{1}{\sqrt{2\left(1+k^{2}\right)}}\left(\begin{array}{l}
k \\
1 \\
1 \\
k
\end{array}\right)
$$

The intersite spin-flip process between sites $m$ and $m+1$ exerts an influence also on sites $m-1$ and $m+2$. This can be expressed by a matrix $\hat{M}_{ \pm}$ which is analogous to $\hat{K}_{ \pm}$

$$
\begin{aligned}
M_{ \pm} & \left(\xi_{m-1}, \xi_{m+2}\right)=2 \alpha^{2} \beta^{2}\left|\alpha_{\xi_{m-1}} \alpha_{\xi_{m+2}}\right| \\
& \cdot\left(O ( 1 , 4 ) O ( 2 , 3 ) \left[O\left(\xi_{m-1}, 1\right) O\left(4, \xi_{m+2}\right) O\left(\xi_{m-1}, 2\right) O\left(3, \xi_{m+2}\right)+\right.\right. \\
& \left.O\left(\xi_{m-1}, 4\right) O\left(1, \xi_{m+2}\right) O\left(\xi_{m-1}, 3\right) O\left(2, \xi_{m+2}\right)\right] \\
\quad+ & O^{2}(1,3) O\left(\xi_{m-1}, 1\right) O\left(3, \xi_{m+2}\right) O\left(\xi_{m-1}, 3\right) O\left(1, \xi_{m+2}\right)+ \\
& \left.O^{2}(2,4) O\left(\xi_{m-1}, 2\right) O\left(4, \xi_{m+2}\right) O\left(\xi_{m-1}, 4\right) O\left(2, \xi_{m+2}\right)\right)
\end{aligned}
$$

which has to be replaced into

$$
\frac{\left\langle\Psi\left|\tau_{m}^{+} \tau_{m+1}^{-}+\tau_{m}^{-} \tau_{m+1}^{+}\right| \Psi\right\rangle}{\langle\Psi \mid \Psi\rangle}=\frac{\left\langle X_{0}\left|\hat{M}_{ \pm}\right| X_{0}\right\rangle}{x_{0}^{3}}
$$


Another useful quantity is the transverse spin polarization which turns out to be the order parameter of the ground state. The matrix belonging to $S_{m}^{x}$ is

$$
\begin{aligned}
\hat{P}_{S}\left(\xi_{m-1}, \xi_{m+1}\right)= & 2 \alpha \beta\left|\alpha_{\xi_{m-1}} \alpha_{\xi_{m+1}}\right| \\
& \cdot\left(O\left(\xi_{m-1}, 1\right) O\left(1, \xi_{m+1}\right) O\left(\xi_{m-1}, 2\right) O\left(2, \xi_{m+1}\right)+\right. \\
& \left.O\left(\xi_{m-1}, 3\right) O\left(3, \xi_{m+1}\right) O\left(\xi_{m-1}, 4\right) O\left(4, \xi_{m+1}\right)\right)
\end{aligned}
$$

while the matrix belonging to $\tau_{m}^{x}$ is the very similar

$$
\begin{aligned}
\hat{P}_{\tau}\left(\xi_{m-1}, \xi_{m+1}\right)= & -2 \alpha \beta\left|\alpha_{\xi_{m-1}} \alpha_{\xi_{m+1}}\right| \\
& \cdot\left(O\left(\xi_{m-1}, 1\right) O\left(1, \xi_{m+1}\right) O\left(\xi_{m-1}, 3\right) O\left(3, \xi_{m+1}\right)+\right. \\
& \left.O\left(\xi_{m-1}, 2\right) O\left(2, \xi_{m+1}\right) O\left(\xi_{m-1}, 4\right) O\left(4, \xi_{m+1}\right)\right)
\end{aligned}
$$

The sign difference in (35) and (36) shows that (as expected for an antiferromagnetic Kondo interaction) the $S$ and $\tau$ polarizations point in opposite directions.

\subsection{The minimization procedure}

Now we take particular forms of the Ansatz and work out the consequences. In the process, we hope to learn which correlations are most relevant for getting a correct description in different regimes of the coupling constant $J / W$.

\subsubsection{Simple spin correlations}

Since the mean field solution gives a magnetic-to-nonmagnetic ground state phase transition, the first idea could be to incorporate short range spin-spin correlations. Though only the $\tau$ spins are subject to intersite interactions, their ordering induces a similar ordering of the $S$-spins. (Since the Kondo coupling is antiferromagnetic, the local $S$-moment is antiparallel to the local $\tau$-moment.) Therefore we incorporate in the Ansatz both $\tau-\tau$ and $S-S$ intersite correlations, controlled by the independent variational parameters $\lambda_{S}$ and $\lambda_{\tau}$. Each antiparallel $\tau-\tau$ pair brings a factor $\lambda_{\tau}$, and each antiparallel $S-S$ pair a factor $\lambda_{S}$. The corresponding matrix of the correlation coefficients 
$O\left(\xi_{i}, \xi_{i+1}\right)$ can be written as

$$
\hat{O}_{1}=\left(\begin{array}{cccc}
1 & \lambda_{S} & \lambda_{\tau} & \lambda_{S} \lambda_{\tau} \\
\lambda_{S} & 1 & \lambda_{S} \lambda_{\tau} & \lambda_{\tau} \\
\lambda_{\tau} & \lambda_{S} \lambda_{\tau} & 1 & \lambda_{S} \\
\lambda_{S} \lambda_{\tau} & \lambda_{\tau} & \lambda_{S} & 1
\end{array}\right)
$$

In principle, we could have included intersite $S-\tau$ correlations as well. Since the motivation for these is not immediately clear, and the minimization difficult enough with the parameters we already have, we omit them.

$\hat{O}_{1}$ which describes fluctuating magnetism, should work reasonably well in the weak-to-intermediate coupling regime where it means an improvement over the mean-field finding of static long-range order.

The largest eigenvalue of the transfer matrix is

$$
\begin{aligned}
x_{0}= & \frac{1}{2}\left[\left(\alpha^{2}+\beta^{2}\right)\left(1+\lambda_{S}^{2} \lambda_{\tau}^{2}\right)+\right. \\
& \left.\sqrt{\left(\alpha^{2}-\beta^{2}\right)^{2}\left(1+\lambda_{S}^{2} \lambda_{\tau}^{2}\right)^{2}+4 \alpha^{2} \beta^{2}\left(\lambda_{S}^{2}+\lambda_{\tau}^{2}\right)^{2}}\right]
\end{aligned}
$$

and $k$ appearing in the corresponding eigenvector

$$
\begin{aligned}
k= & \frac{1}{2 \alpha \beta\left(\lambda_{S}^{2}+\lambda_{\tau}^{2}\right)}\left[\left(\alpha^{2}-\beta^{2}\right)^{2}\left(1+\lambda_{S}^{2} \lambda_{\tau}^{2}\right)^{2}+\right. \\
& \left.\sqrt{\left(\alpha^{2}-\beta^{2}\right)^{2}\left(1+\lambda_{S}^{2} \lambda_{\tau}^{2}\right)^{2}+4 \alpha^{2} \beta^{2}\left(\lambda_{S}^{2}+\lambda_{\tau}^{2}\right)^{2}}\right]
\end{aligned}
$$

These quantities enter the various terms of the ground state energy. Using $\uparrow-\downarrow$ symmetries, the $z-z$ part of the Kondo term can be derived from (26) as

$$
\frac{\left\langle\Psi\left|S_{m}^{z} \tau_{m}^{z}\right| \Psi\right\rangle}{\langle\Psi \mid \Psi\rangle}=\frac{\left(\alpha^{2}-\beta^{2}\right)\left(1+\lambda_{S}^{2} \lambda_{\tau}^{2}\right)}{4 \sqrt{\left(\alpha^{2}-\beta^{2}\right)^{2}\left(1+\lambda_{S}^{2} \lambda_{\tau}^{2}\right)^{2}+4 \alpha^{2} \beta^{2}\left(\lambda_{S}^{2}+\lambda_{\tau}^{2}\right)^{2}}}
$$

For the spin-flip part, we use (31) to arrive at

$$
\frac{\left\langle\Psi\left|S_{m}^{+} \tau_{m}^{-}+S_{m}^{-} \tau_{m}^{+}\right| \Psi\right\rangle}{\langle\Psi \mid \Psi\rangle}=-\frac{2 \beta^{2} \lambda_{S}^{2} \lambda_{\tau}^{2}(\alpha k+\beta)^{2}}{x_{0}^{2}\left(1+k^{2}\right)}
$$

Finally, (34) is used to derive

$$
-\frac{W}{2} \frac{\left\langle\Psi\left|\tau_{m}^{+} \tau_{m+1}^{-}+\tau_{m}^{-} \tau_{m+1}^{+}\right| \Psi\right\rangle}{\langle\Psi \mid \Psi\rangle}=-W \frac{\alpha^{2} \beta^{2} \lambda_{\tau}^{4}\left(1+\lambda_{S}^{2}\right)^{3}(\alpha k+\beta)^{2}}{x_{0}^{3}\left(1+k^{2}\right)}
$$


To get a feeling for the structure of the result, let us first study the case $J=0$ when only the term (42) remains. Maximum freedom for $\tau$-spinflip is obtained for $\alpha=\beta=1 / \sqrt{2}$ which leads to an expression which is independent of $\lambda_{S}:-2 \lambda_{\tau}^{4} /\left(1+\lambda_{\tau}\right)^{2}$. This has its minimum at $\lambda_{\tau}=\sqrt{2}$. The minimum energy $-8 W / 27$ amounts to $94 \%$ of the exact value $-W / \pi$.

For small $J / W$, an expansion in terms of the small quantities $\beta / \alpha-1$, and $t-\sqrt{2}$ yields

$$
\langle H\rangle \approx-\frac{8}{27} W-\frac{2}{9} J-\frac{1}{36} \frac{J^{2}}{W}
$$

which corresponds to $\lambda_{S} \approx 1$ and $\lambda_{\tau} \approx \sqrt{2}(1-3 J / 16 W)$.

For general $J / W$, minimization was carried out numerically. The result for the ground state energy is shown in Fig. 1. In the interval $0 \leq J / W<1$, the ground state energy shows an improvement over the simple mean-field result, which is quite substantial in the small- $J$ regime. However, as $J / W$ is increased slightly beyond 1 , the solution seems to gradually approach that obtained by the single-site mean field treatment.

We confirmed the existence of a sharp phase transition by a semi-analytic argument by expanding the energy in terms of the small parameter $\alpha / \beta$. Strictly for $\alpha=0$, the intersite hopping contribution (42) vanishes, and the Kondo energy has its minimum value $-3 J / 4$ for $\lambda_{S} \lambda_{\tau}=1$. In the neighbourhood of the transition we expect (and find) that $\delta=\lambda_{S}-1 / \lambda_{\tau}$ is also very small, so we can make an additional expansion in terms of $\delta$. The leading terms of the energy can be written as

$$
\langle H\rangle \approx-\frac{3}{4} J+\left[f_{1}\left(J / W, \lambda_{\tau}\right)+f_{2}\left(J / W, \lambda_{\tau}\right) \cdot \delta\right]\left(\frac{\alpha}{\beta}\right)^{2}
$$

$f_{2}$ is negative but an energy lowering due to an infinitesimally small $\delta$ is impossible if $f_{1}$ is a finite positive quantity. We found that the minimum of $f_{1}$ changes sign at $J / W \approx 1.059$. For larger $J$ 's, the total energy increment in non-negative which requires $\alpha=0$, i.e., we are back at the single-site mean field solution.

Below the threshold value, the ground state is ordered, having nonvanishing expectation values of $\tau^{x}$ and $S^{x}$

$$
\left\langle\tau^{x}\right\rangle=-\frac{\alpha \beta \lambda_{\tau}^{2}\left(1+\lambda_{\tau}^{2}\right)^{2}(\alpha k+\beta)^{2}}{x_{0}^{2}\left(k^{2}+1\right)}
$$




$$
\left\langle S^{x}\right\rangle=\frac{\alpha \beta \lambda_{\tau}^{2}\left(1+\lambda_{S}^{2}\right)^{2}(\alpha k+\beta)^{2}}{x_{0}^{2}\left(k^{2}+1\right)}
$$

Our experience with the Ansatz specified by (37) can be summarized like this: introducing independent $\tau-\tau$ and $S-S$ correlations leads to considerable improvement in the description of the ordered state. However, there is still a phase transition from an ordered to a non-ordered ground state at the threshold value $J=1.059 \mathrm{~W}$ which is near to $J=W$ ontained in the ordinary mean field treatment (Fig. 2). For $J>1.059 \mathrm{~W}$, the description reduces to that obtained from the product trial state (8), i.e., it gives an array of decoupled singlets. The description of the high- $J$ regime can be improved by postulating less obvious kinds of intersite correlations.

\subsubsection{Composite spin correlations}

Simple spin correlations failed to provide an acceptable characterization of the ground state for $J / W>1$. To understand the nature of this state, let us remember that in the large $-J$ limit, the process (13) prevents the system from freezing into a collection of singlets: it will keep on creating antiparallel pairs of local triplets. However, these pairs should then dissolve into singlets again, otherwise a high-energy situation remains sustained. Thus there must be a tendency for antiparallel components of local triplets to remain at nearest neighbour distance, which we try to enforce by $O(1,4)=O(4,1)=\eta$. If we wish, we can assist the pair creation of local triplets by enhancing the antiparallel correlations in nearby singlets via $O(2,3)=O(3,2)=\zeta$. Thus we are led to consider

$$
\hat{O}_{2}=\left(\begin{array}{llll}
1 & 1 & 1 & \eta \\
1 & 1 & \zeta & 1 \\
1 & \zeta & 1 & 1 \\
\eta & 1 & 1 & 1
\end{array}\right)
$$

It should be emphasized that here we are considering the correlations of composite objects made up of $S$ - and $\tau$-spins. The correlations we introduced do not factorize into independent $S-S$ and $\tau-\tau$ correlations. 
The largest eigenvalue of the transfer matrix (24) is now

$$
x_{0}=\frac{1}{2}\left(\alpha^{2}\left(1+\eta^{2}\right)+\beta^{2}\left(1+\zeta^{2}\right)+\sqrt{\left[\alpha^{2}\left(1+\eta^{2}\right)-\beta^{2}\left(1+\zeta^{2}\right)\right]^{2}+16 \alpha^{2} \beta^{2}}\right)
$$

which belongs to an eigenvector of the form (32) with

$$
k=\frac{1}{4 \alpha \beta}\left(\alpha^{2}\left(1+\eta^{2}\right)-\beta^{2}\left(1+\zeta^{2}\right)+\sqrt{\left[\alpha^{2}\left(1+\eta^{2}\right)-\beta^{2}\left(1+\zeta^{2}\right)\right]^{2}+16 \alpha^{2} \beta^{2}}\right)
$$

The $z-z$ part of the Kondo coupling is

$$
\frac{\left\langle\Psi\left|S_{m}^{z} \tau_{m}^{z}\right| \Psi\right\rangle}{\langle\Psi \mid \Psi\rangle}=\frac{1}{4} \cdot \frac{\alpha^{2}\left(1+\eta^{2}\right)-\beta^{2}\left(1+\zeta^{2}\right)}{\sqrt{\left[\alpha^{2}\left(1+\eta^{2}\right)-\beta^{2}\left(1+\zeta^{2}\right)\right]^{2}+16 \alpha^{2} \beta^{2}}}
$$

while the spin-flip part is found to be

$$
\frac{\left\langle\Psi\left|S_{m}^{+} \tau_{m}^{-}+S_{m}^{-} \tau_{m}^{+}\right| \Psi\right\rangle}{\langle\Psi \mid \Psi\rangle}=-\frac{2 \beta^{2}(\alpha k+\beta \zeta)^{2}}{x_{0}^{2}\left(1+k^{2}\right)^{2}}
$$

The $\tau$-spin-flip energy is given by

$$
-\frac{W}{2} \frac{\left\langle\Psi\left|\tau_{m}^{+} \tau_{m+1}^{-}+\tau_{m}^{-} \tau_{m+1}^{+}\right| \Psi\right\rangle}{\langle\Psi \mid \Psi\rangle}=-\frac{2 \alpha^{2} \beta^{2}(1+\eta \zeta)[\alpha(1+\eta) k+\beta(1+\zeta)]^{2}}{x_{0}^{3}\left(1+k^{2}\right)^{2}}
$$

The energy expression can be put together from (40), (41), and (42). It has to be minimized with respect to the three independent variational parameters $\alpha / \beta, \eta$, and $\zeta$.

Optimization has to be done numerically and the results are shown in Fig. 3 for the ground state energy and in Fig. 4 for (the absolute value of) the order parameter

$$
\left\langle S^{x}\right\rangle=-\left\langle\tau^{x}\right\rangle=\frac{\alpha \beta[\beta(1+\zeta)+\alpha k(1+\eta)]^{2}}{x_{0}^{2}\left(1+k^{2}\right)}
$$

The most relevant feature is the suppression of the phase transition: the ground state is ordered for all $J$. The ordered moment is of $O(1)$ for weak 
coupling and gets gradually suppressed in the strong coupling regime. We find it intriguing that a hint of reduced moment magnetism emerges in a model which is thought to correspond to the single-channel Kondo lattice.

Details of the behaviour can be discussed in limiting cases:

For $J=0$, only the $x-y$ term remains. Due to the symmetrical role played by local singlets and triplets in enhancing the mobility of the $\tau$ spins the minimum corresponds to $\alpha=\beta$, and $\eta=\zeta$. The lowest value is $\sim-0.281 W$ which we find at $\eta \approx 1.512$. Thus even in the limit which is the exact opposite of what the trial state is intended for, a significant improvement over the mean field solution (energy $-0.25 W$ for $\eta=\zeta=1$, $\alpha=\beta)$ is achieved. The ordered moment is $\left\langle\tau^{x}\right\rangle \approx 0.452$.

For large $J$, the density of local triplets has to be small, meaning $\alpha \ll 1$, while the few triplets that are left must occur in antiparallel pairs so $\eta$ must become large. In contrast, the correlations governed by $\zeta$ become relatively unimportant; for the sake of the present argument, we set $\zeta=1$. Introducing the convenient parametrization $\alpha=\cos \varphi, \beta=\sin \varphi, \Delta \varphi=\pi / 2-\varphi$ is one of the small parameters; the other is $1 / \eta$. Expanding in these, the structure of the energy expression suggests to look for the asymptotic solution in the form

$$
\eta=\frac{c_{1} W}{J(\Delta \varphi)^{2}}
$$

and

$$
\Delta \varphi=\frac{c_{2} W}{J}
$$

where $c_{1}$ and $c_{2}$ are to be determined from optimizing the leading contributions to the energy

$$
\langle H\rangle \approx-\frac{3}{4} J-\frac{W^{2}}{J}\left(\frac{c_{1}}{2}-c_{1}^{2}\right)+\frac{3 c_{1}^{4}}{4(\Delta \varphi)^{2}} \frac{W^{4}}{J^{3}}+J(\Delta \varphi)^{2}
$$

yielding

$$
c_{1}=\frac{\sqrt{3}-1}{8}
$$

and

$$
c_{2}=\sqrt{\frac{\sqrt{3}}{2}} c_{1}
$$


The leading terms of the energy become

$$
\langle H\rangle \approx-\frac{3}{4} J-\frac{\sqrt{3}-1}{32} \cdot \frac{W^{2}}{J}
$$

which is obtained for

$$
\eta \approx 8\left(1+\frac{1}{\sqrt{3}}\right) \cdot \frac{J}{W}
$$

and

$$
\alpha \sim \frac{W}{J}
$$

The asymptotic behaviour of the order parameter is found to be

$$
\left\langle\tau^{x}\right\rangle \approx \frac{3^{1 / 4}(\sqrt{3}-1)}{8 \sqrt{2}} \cdot \frac{W}{J} \cdot\left[1+\frac{(\sqrt{3}-1)}{8} \cdot \frac{W}{J}\right]
$$

Thus our variational method recovered the correct order of magnitude $\sim$ $-W^{2} / J$ of the ground state energy in the large- $J$ limit: it is what we would expect from perturbation theory. The nature of the problem is similar to that of the large $-U$ behaviour of the Hubbard model where nearest-neighbour holon-doublon correlations were found to be important [17].

In retrospect we can identify the reason why simple spin correlations are insufficient in the large $-J$ regime. The correlation matrix (37) enforces $O(1,4)=O(2,3)$ while with (47) we have found that $O(1,4) / O(2,3)$ has to become very large as $J / W$ increases.

\section{Discussion and conclusion}

We were trying to achieve a more detailed understanding of the behaviour of the Kondo necklace model, with the eventual aim of finding results which may be relevant to the physics of heavy fermion systems. We were considering the necklace hamiltonian (11) with $\rho=0$, i.e., the simplest form introduced by Doniach [9].

The single-site mean field solution of (1) indicates that with increasing $W / J$, a magnetic-to-Kondo (ground state) phase transition is taking place.

While this is a physically appealing result, the obvious shortcomings of the 
characterization of the high- $J$ state as an array of disconnected singlets may lead to worries that the phase transition is merely an artefact of the approximation.

We improved the variational description of the ground state by allowing for the presence of a variety of nearest-neighbour correlations. We carried out the optimization in the one-dimensional case where the transfer matrix method [16] can be used to calculate the relevant expectation values.

In Section 3.3.1 we used the simple spin correlations whose presence can be inferred from the ordering. These led to a better ground state energy in the small-to-intermediate $J$ regime, and pushed the phase transition point slightly upwards (Figures 1 and 2). However, the high- $J$ state remained as structureless as in the simplest mean field approximation.

In Section 3.3.2 we learned that the physically interesting ones are the composite spin correlations which could be expressed as expectation values of products of four spin-operators, involving both kinds of spins (we could choose $\left.\left\langle S_{i}^{+} \tau_{i}^{+} S_{i+1}^{-} \tau_{i+1}^{-}\right\rangle\right)$. The relevance of these could be guessed from perturbation theory: they describe that for $J \gg W$, the ground state is almost singlet, with a sprinkling of a few nearest-neighbour antiparallel triplet pairs. The inclusion of these correlations suppresses the phase transition completely (Figures 3 and 4), the ground state remains ordered for any finite $J$. Furthermore, the ground state energy, and the concentration of local triplets have the order of magnitude expected from perturbation theory. The tail regime of the order parameter (Fig. 4) is a tantalizing hint that small ordered moments may be a part of the physics of the orbitally non-degenerate Kondo lattice.

However, we have to be extremely cautious about the conclusions to be drawn from our results. After all, for the one-dimensional Kondo necklace, powerful techniques have provided a number of essentially exact statements, and these tend to be in disagreement with our findings. General arguments suggest [7] that for almost the entire range of $J / W$ values, the excitation spectrum is gapped but at some small $J / W$, the possibility of a phase transition can not be excluded. In fact, exact diagonalization studies [18] revealed the existence of a Kosterlitz-Thouless type transition from the gapped high$J$ state (with exponential decay of spin correlations) to a gapless low- $J$ state (with algebraic decay of spin correlations). In any case, the ground state never has true long-range order.

This point in itself should not be too worrying. Our correlated mean 
field approach is supposed to work where long-range order is in principle possible, i.e., foremost in three dimensions. It is just technical convenience which made us to stick to one dimension but we could argue that the general appearance of our results is the same as what a much more cumbersome three-dimensional evaluation should give. This would still allow us to hope that the three-dimensional pseudospin model would have a ground state with small ordered moments.

We have to be, however, aware of a subtler kind of difficulty as well. Prescribing an Ansatz means that the system is permitted to seek a low-energy state in a certain manner. This can lead to a good estimate for the ground state energy (as it undoubtedly does) but does not necessarily imply that this is the natural way how the energy gain in question arises. To cite an example, a similar study of the Hubbard model [17] gave the correct order of magnitude $-t^{2} / U$ for the energy at $U \gg t$ but ascribed it to a metallic ground state which is patently false. Hence we should be warned that finding the (functionally) correct asymptotic form (59) of the ground state energy does not prove that the result (62) about the long-range order is basically right. In fact, preliminary results 19 obtained from the Oguchi approximation indicate that the order parameter $\left\langle\tau^{x}\right\rangle$ vanishes above a critical $J / W$. It remains an outstanding question whether the three-dimensional Doniach pseudospin model can support reduced moment magnetism.

\section{ACKNOWLEDGEMENTS}

The authors wish to express their gratitude to the International Centre for Theoretical Physics for financial support, hospitality, and an encouraging scientific atmosphere. P.F. is indebted also to SISSA (Trieste) for the hospitality extended to him.

\section{References}

[1] For reviews of the physics of $f$-electron systems see, e.g., P.A. Lee, T.M. Rice, J.W. Serene, L.J. Sham and J.W. Wilkins: Comments Cond. Matt. Phys. XII, 99 (1986), and the more recent work by N. Grewe 
and F. Steglich, in: Handbook on the Physics and Chemistry of the Rare Earths Vol. 14, Ed. K.A. Gschneidner, Jr. and L. Eyring, (NorthHolland, Amsterdam, 1991) pp. 434-474.

[2] P. Coleman and J. Gan: Physica B171, 3 (1991).

[3] T.M. Rice and K. Ueda: Phys. Rev. B34, 6420 (1986).

[4] P. Fazekas: Solid State Commun. 60, 431 (1986).

[5] H. Shiba and P. Fazekas: Progr. Theor. Phys. Suppl. No. 101, 403 (1990).

[6] P. Fazekas and H. Shiba: Int. J. Modern Phys. B5, 289 (1991).

[7] S.P. Strong and A.J. Millis: Phys. Rev. Lett. 69, 2419 (1992).

[8] V.L. Líbero and D.L. Cox: preprint.

[9] S. Doniach: Physica B91, 231 (1977).

[10] P. Fazekas and E. Müller-Hartmann: Z. Physik B85, 285 (1991).

[11] H. Tsunetsugu, Y. Hatsugai, K. Ueda, and M. Sigrist: Phys. Rev. B46, 3175 (1992); T. Nishino and K. Ueda: unpublished.

[12] J.W. Rasul and P. Schlottmann: Phys. Rev. Lett. 62, 1701 (1989); B.A. Jones and C.M. Varma: Phys. Rev. Lett. 62, 1702 (1989).

[13] P. Fazekas and H.Y. Kee: Modern Phys. Lett. B 6, 1681 (1992).

[14] P. Fazekas and H.Y. Kee: Phys. Rev. B (in press).

[15] E. Lieb, Th. Schultz, and D. Mattis: Ann. Phys. 16, 407 (1961).

[16] A. Virosztek: J. Phys. C18, 4735 (1985).

[17] P. Fazekas and K. Penc: Int. J. Modern Phys. B2, 1021 (1988).

[18] P. Santini and J. Sólyom: Phys. Rev. B46, 7422 (1992).

[19] H.Y. Kee, S. Kim, and P. Fazekas: unpublished. 


\section{Figure captions}

Fig. 1. Ground state energy (in units of $W$ ) versus $J / W$ for a trial state with intersite $S-S$ and $\tau-\tau$ spin correlations. Thick line: correlated mean field solution (transition at $J / W \approx 1.059$ ), thin line: simple mean field (transition at $J / W=1$ ).

Fig. 2. The order parameter $\left\langle\tau^{x}\right\rangle$, for the same case as in Fig. 2.

Fig. 3. Ground state energy (in units of $W$ ) for a trial state with composite intersite correlations, belonging to the antiparallel alignment of fluctuating triplets. Thick line: correlated mean field, thin line: simple mean field.

Fig. 4. Order parameter $\left\langle\tau^{x}\right\rangle$ versus $J / W$ for the same case as Fig. 3. The ground state phase transition found in the simple mean field solution (thin line) is suppressed, according to the correlated mean field approach (thick line), the asymptotic behaviour is $\sim W / J$. 